\title{
Asymptotic of the electric structure function and the deuteron wave function
}

\author{
V. I. Zhaba \\ Uzhgorod National University, Department of Theoretical Physics, \\ 54, Voloshyna St., Uzhgorod, UA-88000, Ukraine \\ E-mail:viktorzh@meta.ua
}

\begin{abstract}
The main features of obtaining the asymptotic behaviour of the electric structure function $A(p)$ at large values of the transmitted momentum are analysed. The asymptotic behaviour of the structure function $A(p)$ was determined to take into account the asymptotic behaviour of the deuteron form factors and the original dipole approximation for the nucleon form factors. Asymptotic values of $A(p)$ were obtained for the nucleon-nucleon potential Reid93 and compared with the calculations for different nucleon form factors models and their approximations. In the broad momentum range up to $12.5 \mathrm{fm}^{-1}$, the basic forms of the asymptotic behaviour of the electric structure function are demonstrated and compared with the experimental data of the modern collaborations. As the analysis shows in most cases considered, the asymptotic for $A(p)$ is represented in the form of the power function $p^{-n}$.

Абстракт Проаналізовано основні особливості одержання асимптотичної поведінки функції електричної структури $A(p)$ при великих значеннях переданого імпульсу. Асимптотична поведінка структурної функції $A(p)$ була визначена при врахуванні асимптотичної поведінки дейтронних формфакторів та оригінального дипольного наближення для нуклонних формфакторів. Для нуклон-нуклонного потенціалу Reid93 одержано асиптотичні значення $A(p)$ i порівняно їх із розрахунками для різних моделей нуклонних формфакторів та їх наближень. В широкому діапазоні імпульсів до $12.5 \mathrm{fm}^{-1}$ продемонстровано основні форми асимптотичної поведінки функції електричної структури та порівняно їх з експериментальними даними провідних колаборацій. Як показує аналіз, у більшості розглянутих випадків асимптотика для $A(p)$ представлена у формі степеневої функції $p^{-n}$.
\end{abstract}

Keywords: structure function, asymptotic, deuteron, form factor, approximation, wave function.

PACS: 13.40.Gp, 13.88.+e, 21.45.Bc, 03.65.Nk

\section{Introduction}

As a two-nucleon bound state, the deuteron is the simplest system in nuclear physics. This simple two-particle system allows us to better understand the nucleon-nucleon interaction in the processes of deuteron scattering. Measurements of the electromagnetic properties of a deuteron are invaluable for studying the internal structure of a deuteron.

The electromagnetic structure of the deuteron is observed in the elastic scattering of electrons on deuterons and can be described by the three form factors $G_{C}, G_{Q}$ and $G_{M}$, which are the electric monopole, the electric quadrupole and the magnetic dipole distributions of the deuteron respectively in transferred momentum squared $p^{2}$ representation [1]. From measurements of unpolarized elastic electron-deuteron cross-section at different scattering angles, two combinations of the deuteron form factors $A(p)$ and $B(p)$ can be obtained (by Rosenbluth separation).

The deuteron elastic structure function $A(p)$ can be extracted from cross-section measurements of elastic scattering of electrons on deuterons in coincidence (for example, in the range $0.7 \leq p^{2} \leq 6.0$ $(\mathrm{GeV} / \mathrm{c})^{2}$ for Hall A of Jefferson Laboratory [2]). At low values of momentum $p^{2}$, the cross-section is dominated by $A(p)$, and in $A$ is dominated by form factor $G_{C}$ [1]. One of the latest experimental 
measurements for the electric structure function $A(p)$ was presented in an intermediate momentum transfer region in [3] and for large momentum in [2, 4,. The experimental status of $A(p)$ is described in detail in the review [5].

We note another important feature of the knowledge of the electric structure function $A(p)$. It is a component in tensor $t_{2 j}$ and vector $t_{1 i}$ polarizations [5], in the spin correlation coefficients and tensor asymmetries [6], and other polarization observables in processes with the participation of deuteron. The value $A(p)$ appears in the factor $S\left(p, \theta_{e}\right)=A(p)+B(p) \operatorname{tg}^{2}\left(\theta_{e} / 2\right)$, where $\theta_{e}$ is the electron scattering angle in the laboratory system.

A theoretical studies of the value $A(p)$ in ed-scattering using modern theoretical approaches and approximations remains relevant [7, 8, 9, 10, 11. In this paper, we use the analytic forms of the deuteron wave function (DWF) in coordinate representation for theoretical calculations of electric structure function $A(p)$ and its asymptotic at large momentums in the elastic electron-deuteron scattering. The nucleon-nucleon realistic phenomenological potential of Nijmegen group Reid93 [12, 13, 14] and the different models of nucleon form factors were used for numerical calculations.

\section{The electric structure function}

Different models of NN potential for quantitative understanding of the structure of the deuteron, S- and D-states and polarization characteristics are considered. The deuteron charge distribution is not well known from the experiment because it is determined from the data of polarization experiments (polarizations and differential cross-sections) [15]. Differential cross-section of elastic scattering of unpolarized electrons by unpolarized deuterons without measuring the polarization of reflected electrons and deuterons is given by the formula within the assumptions of the first Born approximation and the conditions of relativistic invariance [16, 17, 18, 19, 5]

$$
\frac{d \sigma}{d \Omega_{e}}=\left(\frac{d \sigma}{d \Omega_{e}}\right)_{M o t t}\left[A\left(p^{2}\right)+B\left(p^{2}\right) \operatorname{tg}^{2}\left(\frac{\theta_{e}}{2}\right)\right] .
$$

Formula $[1]$ is obtained by Rosenbluth [20]. Here $\left(\frac{d \sigma}{d \Omega}\right)_{\text {Mott }}$ is the scattering cross-section on a spinless structureless particle obtained by Mott; $p$ is the transmitted deuteron momentum in units fm-1; $A(p)$ and $B(p)$ are the electric and magnetic structure functions (or structure functions determined by the electromagnetic structure of a deuteron)

$$
\begin{gathered}
A=G_{C}^{2}+\frac{8}{9} \eta^{2} G_{Q}^{2}+\frac{2}{3} \eta G_{M}^{2} \\
B=\frac{4}{3} \eta(1+\eta) G_{M}^{2},
\end{gathered}
$$

where $\eta=\frac{p^{2}}{4 m_{d}^{2}} ; m_{d}$ - is the mass of deuteron. The charge $G_{C}(p)$, quadrupole $G_{Q}(p)$ and magnetic $G_{M}(p)$ deuteron form factors (FFs) contain information about the electromagnetic characteristics of a deuteron [21, 22, 23, 24, 25, 5, 26]:

$$
G_{C}=G_{E N} D_{C} ; \quad G_{Q}=G_{E N} D_{Q} ; \quad G_{M}=\frac{m_{d}}{2 m_{p}}\left(G_{M N} D_{M}+G_{E N} D_{E}\right)
$$

The body form factors $D_{i}$ are determined by DWFs in the coordinate representation monopoly electric $D_{C}=\int_{0}^{\infty}\left[u^{2}+w^{2}\right] j_{0} d r$

quadrupole electric $D_{Q}=\frac{3}{\sqrt{2} \eta} \int_{0}^{\infty}\left[u w-\frac{w^{2}}{\sqrt{8}}\right] j_{2} d r$

transverse magnetic $D_{M}=2 \int_{0}^{\infty}\left[\left(u^{2}-\frac{w^{2}}{2}\right) j_{0}+\left(\frac{u w}{\sqrt{2}}+\frac{w^{2}}{2}\right) j_{2}\right] d r$; 
longitudinal magnetic $D_{E}=\frac{3}{2} \int_{0}^{\infty} w^{2}\left[j_{0}+j_{2}\right] d r$;

where $G_{E N}=G_{E p}+G_{E n} ; G_{M N}=G_{M p}+G_{M n}$ are the isoscalar electric and magnetic FFs; $G_{E p}$ and $G_{E n}$ are proton and neutron isoscalar electric FFs; $G_{M p}$ and $G_{M n}$ are proton and neutron isoscalar magnetic FFs; $j_{0}, j_{2}$ are spherical Bessel functions of zero and second order from argument $\operatorname{pr} / 2$.

The original dipole fit $(\mathrm{DFF})$ is the simplest representation for the proton and neutron FFs [27, 28]:

$$
G_{E p}=F_{N} ; \quad G_{E n}=0 ; \quad G_{M p}=\mu_{p} G_{E p} ; \quad G_{M n}=\mu_{n} G_{E p}
$$

Here the nucleon $\mathrm{FF}$ is written in the form of a dipole [27]

$$
F_{N}\left(p^{2}\right)=\left(1+\frac{p^{2}}{0.71(G e V / c)^{2}}\right)^{-2}=\left(1+\frac{p^{2}}{18.235 f m^{-2}}\right)^{-2} .
$$

Analysing formulas (1)-(3), it will be obvious [29] that the angular dependence of the differential cross-section allows us to independently measure the structure functions $A$ and $B$. However, experiments for unpolarized particles do not give complete information about all quantities describing ed-scattering and not allow dividing the contributions of charge and quadrupole FFs in to $A$. Therefore, additional experiments with polarized particles are required for a complete description: 1) scattering on a polarized deuteron target; 2) measurement of tensor polarization of recoil deuterons.

Experimental data for the electric structure function $A(p)$ are given in the papers of Stanford [30], Orsay [31, 32], CEA [16], DESY [17], SLAC [33, 34], Mainz [35], Bonn [36], Saclay [37], Bates 38], JLab 3, 2], JLab2007 [4] collaborations and in Garcon review [38].

\section{Asymptotic of the electric structure function}

In quantum chromodynamics (QCD) [39, 11], with large momentums, asymptotic values and their relations for the structure functions and for deuteron FFs are written as:

$$
\begin{gathered}
\sqrt{A} \sim \sqrt{B} \sim G_{C} \sim \frac{1}{p^{10}} ; \quad G_{Q} \sim G_{M} \sim \frac{1}{p^{12}} ; \quad B: A: G_{C}^{2}=4: 1: \frac{1}{3} ; \\
G_{C}: G_{M}: G_{Q}=\left(1-\frac{2}{3} \eta\right): 2:-1 .
\end{gathered}
$$

In addition to the representation of the structure functions $A$ and $B$, in paper [40] there is a form of presentation of the results in the form $A\left(p^{2}\right) /\left(F_{N}^{2} F\right)$ and $B\left(p^{2}\right) /\left(F_{N}^{2} F_{1}\right)$, and also according to [41] in the form as $\Gamma_{M}\left(p^{2}\right) /\left(F_{N}^{2} F_{1}\right)$, where $\Gamma_{M}\left(p^{2}\right)=\left[G_{M}\left(p^{2}\right) m_{p} /\left(\mu_{d} m_{d}\right)\right]^{2} ; F=\left(1+p^{2} / 0.1\right)^{-2.5}$; $F_{1}=\left(1+p^{2} / 0.1\right)^{-3}$. Values $F$ and $F_{1}$ in dimensions $\left[(\mathrm{GeV} / \mathrm{c})^{2}\right]$ for $p^{2}$. Determination of structure function $A(p)$ is important for the study of the deuteron $\mathrm{FF}$

$$
F_{d}\left(p^{2}\right)=\sqrt{A\left(p^{2}\right)}
$$

and of the reduced deuteron FF [42]

$$
f_{d}\left(p^{2}\right)=F_{d}\left(p^{2}\right) / F_{N}^{2}\left(\frac{p^{2}}{4}\right) .
$$

The deuteron FF in QCD is defined as 43

$$
F_{d}\left(p^{2}\right) \approx\left[\frac{\alpha_{S}\left(p^{2}\right)}{p^{2}}\right]^{5} \sum_{m, n} d_{m n}\left[\ln \left(\frac{p^{2}}{\Lambda^{2}}\right)\right]^{-\gamma_{n}^{d}-\gamma_{m}^{d}}\left[1+O\left(\alpha_{S}\left(p^{2}\right) ; \frac{m}{p}\right)\right]
$$


In the predictions of QCD [43], the reduced FF at $p^{2}>2(\mathrm{GeV} / \mathrm{c})^{2}$ has the following asymptotic

$$
f_{d}\left(p^{2}\right) \approx \frac{\alpha_{S}\left(p^{2}\right)}{p^{2}}\left[\ln \left(\frac{p^{2}}{\Lambda^{2}}\right)\right]^{-\lambda}
$$

where $\lambda=\frac{2}{5} \frac{C_{F}}{\beta}=-\frac{8}{145} ; C_{F}=\frac{N_{c}^{2}-1}{2 N_{c}} ; \beta=11-\frac{2}{3} N_{f}$. Here $N_{c}=3$ and $N_{f}=2$ are the numbers of colours and flavors of quarks.

It was shown in [43] that the structure function (2) can be decomposed by the nucleon FF $F_{N}\left(p^{2} / 4\right)$ and the so-called "reduced"nuclear FF $f_{d}\left(p^{2}\right)$ :

$$
\sqrt{A\left(p^{2}\right)}=f_{d}\left(p^{2}\right) F_{N}^{2}\left(p^{2} / 4\right)
$$

Parameterization was used to determine the reduced deuteron FF as [44]

$$
f_{d}\left(p^{2}\right)=N \frac{\alpha_{S}\left(p^{2}\right)}{p^{2}}\left[\ln \left(\frac{p^{2}}{\Lambda^{2}}\right)\right]^{-\Gamma},
$$

where $N$ is the normalization factor; $\alpha_{S}\left(p^{2}\right)=\left[\ln \left(\frac{p^{2}}{\Lambda^{2}}\right)\right]^{-1}$ are strong interaction coupling constant; $\Lambda$ is the QCD-scale parameter; $\Gamma=-8 / 145$ is determined by the leading anomalous dimension.

The asymptotic for the deuteron FF at large momentums are determined by the expression [45]:

$$
F_{d}\left(p^{2}\right) \sim \frac{1}{\left(p^{2}\right)^{m}}
$$

where $m=7 / 2$ and $13 / 4$ are for non-relativistic and relativistic impulse approximations (NRIA and RIA) respectively. In addition, in paper [46] it is noted that the magnetic FF $G_{M}$ makes a major contribution to the structure function $A(p)$ and, accordingly, to the deuteron FF $F_{d}$ (in the non-relativistic IA):

$$
A^{(N R)}\left(p^{2}\right) \sim \frac{1}{\left(p^{2}\right)^{6}} \frac{\left(\mu_{p}+\mu_{n}\right)^{2}\left(m_{0}^{2}\right)^{4}}{3 \pi^{2} m_{d}^{2}} \frac{\left[\sum_{j} C_{j} m_{j}^{2}\right]^{2}\left[\sum_{j} C_{j} / m_{j}^{2}\right]^{4}}{\left[\sum_{j} C_{j} / m_{j}^{4}\right]^{2}} ; \quad F_{d}^{(N R)}\left(p^{2}\right) \sim \frac{1}{\left(p^{2}\right)^{3}} ;
$$

where $m_{0}^{2}=0.71(\mathrm{GeV} / \mathrm{c})^{2} ; C_{j}, m_{j}$ are the expansion coefficients of the S-component of DWF in the momentum representation. In the RIA, the asymptotics for $A$ and $F_{d}$ are defined as follows [46]:

$$
A^{(R)} \sim \frac{m_{d}}{p} A^{(N R)} ; \quad F_{d}^{(R)} \sim \frac{F_{d}^{(N R)}}{\sqrt{p}} .
$$

In addition, in [47] takes into account the influence of the "non-Rosenbluth" behaviour of proton FFs on deuteron FFs and, as a consequence, the major contribution to the asymptotic of the structure function $A(p)$ gives the magnetic deuteron FF and the "non-Rosenbluth" behaviour of proton FFs enhances this effect. The obtained asymptotics [47] for $A$ and $F_{d}$ in NRIA (NR) and RIA (R) are written as

$$
A^{(N R)}\left(p^{2}\right) \sim \frac{1}{\left(p^{2}\right)^{8}} \frac{2048\left(\mu_{p}+\mu_{n}\right)^{2}\left(m_{0}^{2}\right)^{4}}{3 \pi m_{d}^{2}}\left[\sum_{j} C_{j} m_{j}^{2}\right]^{4} ; \quad F_{d}^{(N R)}\left(p^{2}\right) \sim \frac{1}{\left(p^{2}\right)^{4}}
$$




$$
A^{(R)} \sim \frac{p^{3}}{32 m_{d}^{3}} A^{(N R)} ; \quad F_{d}^{(R)} \sim p^{3 / 2} F_{d}^{(N R)} .
$$

As stated in [47, given the experimental data JLab [3, 2] for structure function $A(p)$ for momentum values of $p^{2} \sim 6(\mathrm{GeV} / \mathrm{c})^{2}$, the deuteron FF can be interpolated by the function

$$
F_{d}^{(\exp )} \sim \frac{1}{\left(p^{2}\right)^{3.76 \pm 0.41}} .
$$

The perturbative QCD (pQCD) is used to calculate the rate of fall of deuteron FFs for large values $p^{2}$ [48], where for a structure function (2) is valid the approximation $A\left(p^{2}\right) \sim c_{0} / p^{2 n}$ at $n=9$; 10.

The deuteron FF was obtained in the high-quality description of the deuteron electromagnetic FFs in a soft-wall anti-de Sitter/quantum chromodynamics approach (AdS/QCD) as [49]

$$
F_{d}\left(p^{2}\right) \equiv F\left(p^{2}\right)=\frac{\Gamma(6) \Gamma(a+1)}{\Gamma(a+6)},
$$

where $F$ is the twist- 6 hadronic form factor; $a^{2}=p^{2} /\left(4 \kappa^{2}\right)$; the scale parameter $\kappa$ in the range of $150 \mathrm{MeV}<\kappa<250 \mathrm{MeV}$.

\section{Calculations and conclusions}

One of the simplest and most convenient analytical forms of DWFs in coordinate representation is the following [50]

$$
\left\{\begin{array}{l}
u(r)=r \sum_{i=1}^{N} A_{i} e^{-a_{i} r} \\
w(r)=r^{3} \sum_{i=1}^{N} B_{i} e^{-b_{i} r} .
\end{array}\right.
$$

The number of expansions in $(22)$ for Reid93 potential was $N=29$ since in this case, the calculated deuteron parameters by these forms will be good (deuteron radius $r_{m}$, electric quadrupole moment, magnetic moment, the D-state probability, asymptotic D/S-states, etc.).

In addition to the simplest DFF according to (5), the calculations of the structure function $A(p)$ for other theoretical models and the approximations for the nucleon form factors were performed. The first group includes the following: the modified dipole fit 2 (labelled as MDFF2) [28], the relativistic harmonic oscillator model $(\mathrm{RHOM})$ based on the quark model with the relativistic oscillator potential [51, 52, the vector meson dominance model (VMDM) [53] taking into account the parameters of the full Gari-Krumpelmann model [54]; and the second - Kelly parameterization (Kelly) [55], the approximation based JLab measurements of the isoscalar electric and magnetic FFs (JLab) [5] and Bradford parameterization (Bradford) [56].

Fig. 1 shows the results of the calculations of the electric structure function $A(p)$ by selecting seven specified sets of nucleon form FFs and applying of DWF (22) for the nucleon-nucleon potential Reid93. Theoretical calculations in comparison with experimental data [30, 31, 32, 16, 17, 33, 34, 35, 36, 37, 38, 3, 2, 4, 38. The best agreement of the theoretical results with the experiment is available for calculations when choosing VMDM for momentums up $p=13 \mathrm{fm}^{-1}$, and for approximations, 
there will be coincidence only for momentum at $p \leq 5 \mathrm{fm}^{-1}$.

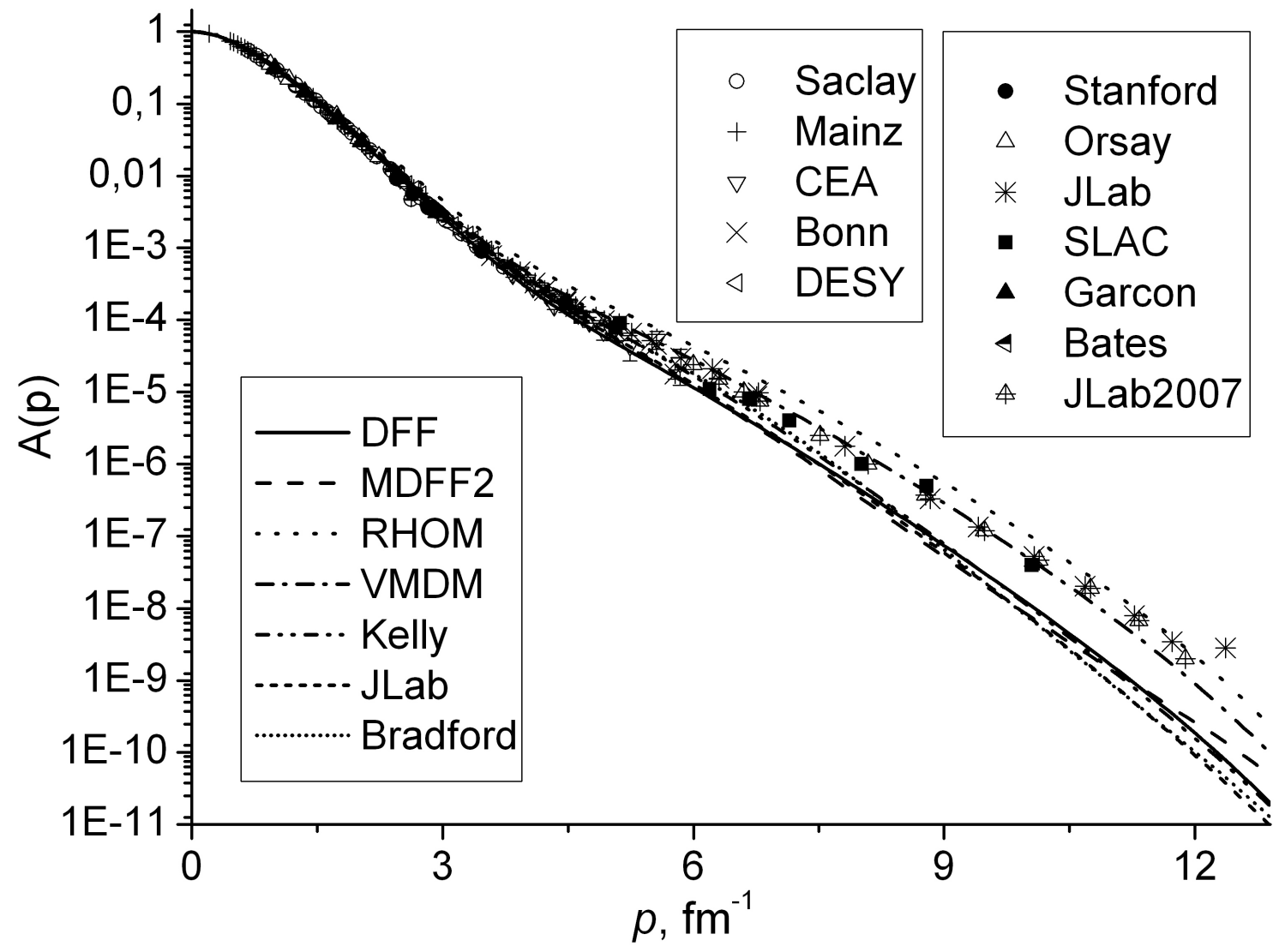

Fig. 1. The electric structure function $A(p)$. The theoretical calculations for DWF (22) with models [27, 28, 51, 52, 53, 54, 55, 5, 56] are compared with the experimental data from [30, 31, 32, 16, 17, 33, 34, 35, 36, 37, 38, 3, 2, 4, 38,

In [57] for DWFs (22) obtained the deuteron FFs $G_{i}$, which is determined by the coefficients of the analytical forms for DWFs, nucleon isoscalar FFs and the order of momentum

$$
\begin{gathered}
G_{C} \approx 32 G_{E N} \sum_{i, j=1}^{N} \frac{A_{i} A_{j} a_{i j}}{\left(p^{2}+4 a_{i j}^{2}\right)^{2}} ; \quad G_{Q} \approx-\frac{9216 G_{E N}}{\sqrt{2} \eta} \sum_{i=1}^{N} \frac{A_{i} B_{i}\left(a_{i}+b_{i}\right) p^{2}}{\left(p^{2}+4\left(a_{i}+b_{i}\right)^{2}\right)^{4}} \\
G_{M} \approx \frac{m_{d}}{m_{p}} G_{M N}\left(32 \sum_{i, j=1}^{N} \frac{A_{i} A_{j} a_{i j}}{\left(p^{2}+4 a_{i j}^{2}\right)^{2}}-\frac{3072}{\sqrt{2}} \sum_{i=1}^{N} \frac{A_{i} B_{i}\left(a_{i}+b_{i}\right) p^{2}}{\left(p^{2}+4\left(a_{i}+b_{i}\right)^{2}\right)^{4}}\right) .
\end{gathered}
$$

where $a_{i j}=a_{i}+a_{j} ; b_{i j}=b_{i}+b_{j}$. And, substituting in (23) and (24) the values of $G_{E N}, G_{M N}$ (for DFF) and $\eta$, we can write the asymptotics of the deuteron FFs for large momentum values in the form [57]:

$$
G_{C} \sim \frac{1}{p^{8}} ; \quad G_{Q} \sim \frac{1}{p^{12}} ; \quad G_{M} \sim \frac{1}{p^{8}} .
$$

In formulas (25) takes into account only the leading parts at large momentum and the simplification of records without the expansion coefficients of DWF (22). When using asymptotics of deuteron FFs at large momentums (25), we find the asymptotic of the electric structure function (2) as $A \sim\left(\frac{1}{p^{8}}\right)^{2}+\eta^{2}\left(\frac{1}{p^{12}}\right)^{2}+\eta\left(\frac{1}{p^{8}}\right)^{2}$, which will be determined by the third part, that is, the magnetic FF: 


$$
A \sim \frac{1}{p^{14}}
$$

The exact value of asymptotic for $A(p)$ can be obtained by considering expressions (23) and (24).

Comparison of our asymptotic (26) for DWFs (22) with other asymptotics (15)-(19) in NRIA and RIA [46, 47] for which $A \sim \frac{1}{p^{k}}$ (at $k=12 ; 13 ; 16 ; 13$ respectively) indicates their similarity.

According to the approximation [47] according to formula (20) for the electric structure function $A(p)$ is a fair expression $\sqrt{A^{(\text {approx } 1)}} \sim \frac{1}{\left(p^{2}\right)^{3.76}}$. The approximation of the experimental data of the JLab [2] and JLab2007 [4] collaborations in the momentum interval at $p \approx 10.6-12.4 \mathrm{fm}^{-1}$ using the function $\frac{1}{\left(p^{2}\right)^{N}}$ similar to 15 gives the result for $N \approx 3.795575$, i.e. $\sqrt{A^{(\text {approx } 2)}} \sim \frac{1}{\left(p^{2}\right)^{3.795}}$. In Fig. 2 , the results of these two approximations are labelled as approx1 and approx2 respectively, and the calculations using the DWF 22 for Reid93 potential at different nucleon FFs are indicated as lines.

The approximation of the calculated values $A(p)$ in the range momentums at $p=10.6-12.5 \mathrm{fm}^{-1}$ using the function $p^{-N}$ showed, that the value $N$ was obtained as 8.44, 8.50, 7.48, 7.82, 8.50, 8.68 and 8.69 for DFF, MDFF2, RHOM, VMDM, JLab, and Bradford respectively. That is, closer to the experimental approximation are the values for RHOM and VMDM.

To estimate the quality of the approximation, the value $\chi^{2}$ is calculated, which per degree of freedom of function. Compared to the experiment, the value $\chi^{2}$ (multiplied by a factor $10^{-16}$ ) for approx1, approx2, RHOM, VMDM is 1.21, 1.63, 3.46, 0.27 respectively. Obviously, the "best" approximation is approx1, and of the two variants the RHOM model is "worse".

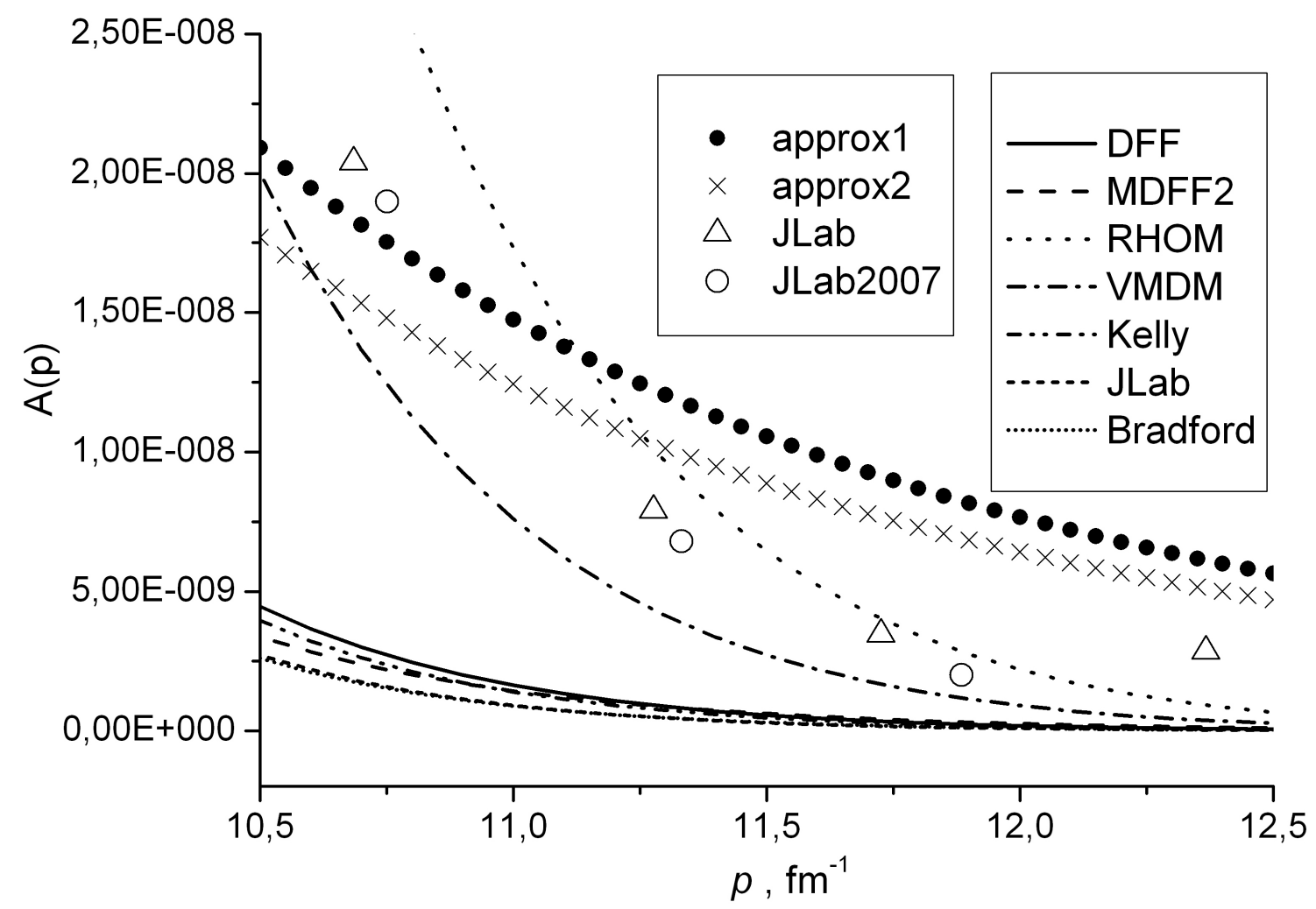

Fig. 2. The electric structure function $A(p)$ at large values of the momentum. The theoretical calculations for DWF (22) with models [27, 28, 51, 52, 53, 54, 55, 5, 56] are compared with the experimental data from [2, 4] and with the two approximations

As can be seen from Figs. 1 and 2 almost all (except for the results for RHOM) the theoretical values obtained for the electric structure function $A(p)$ are below the experimental data. Similar 
behaviour of $A(p)$ was obtained in [46, 47] for NRIA and RIA, for Moscow, NijmI, NijmII, CDBonn, Paris potentials in [10], for calculations in chiral effective field theory [9] at leading order (LO) and with inclusion of charge operators up to $\mathrm{N}^{3} \mathrm{LO}$, for NRIA [26] predictions using non-dipole and dipole behaviour $G_{E p}(p)$, for phenomenological potentials in NRIA (Bonn-A, B, C, Q, Reid$\mathrm{SC}$, Paris and Argonne v18 at $p>4 \mathrm{fm}^{-1}$ [19]), for generalized IA [58] at $p>5 \mathrm{fm}^{-1}$, for equal time approximation and relativistic quasipotential approximation of the Blankenbecler-Sugar-LogunovTavkhelidze [59].

Below the experimental data are the calculations $A(p)$ [60] (at $\left.p>3.6 \mathrm{fm}^{-1}\right)$ for Reid93 potential using the Gari-Krumpelmann and Mergell-Meissner-Drechsel parameterizations of the nucleon FFs.

As the analysis shows, above the experimental data are the values $A(p)$, that were calculated for OBEPQ-A, B, C potentials [61] (for momentum values at $p>3 \mathrm{fm}^{-1}$ ), for IA with the $\rho \pi \gamma$ MEC included [62] (at $p>9 \mathrm{fm}^{-1}$ ), for Bonn (Bonn-B, FULLF, OBEPF) and Nijmegen (Nijm93, NijmI, NijmII) potentials of the Gari-Krumpelmann nucleon FFs [63] (at $\left.p>4 \mathrm{fm}^{-1}\right)$.

For the momentum interval at 7.2-13 $\mathrm{fm}^{-1}$, two approximations for $A(p)$ were performed in [19]. The first approximation is obtained for dimensional scaling in pQCD [64], wherein the case of a hadron composed of $n$ quarks, one can expect that the leading FF has asymptotic as $p^{-2(n-1)}$. Since this true in both the initial and final states, so the FF of the system is written as $F^{2} \sim p^{-4(n-1)}$, and for deuteron $(n=6)$ the asymptotic for the structure function will be as $A \sim p^{-20}$. The second approximation in [19] is as $A \sim p^{-16}$ follows. The fit of the five data for the highest $p^{2}$ from [2] using $A=p^{-2 m}$ gives the value $m=8.0$. If we exclude the inaccurate last point, then $m=8.7$. The experimental data point [2] at $5.955(\mathrm{GeV} / \mathrm{c})^{2}\left(\right.$ or $\left.12.37 \mathrm{fm}^{-1}\right)$ does not lie on the approximation dependence $A=p^{-2 m}$ at $m=8.0$. All curves in paper [19] (see Fig. 5.17) are normalized to the point at $p^{2}=11.275 \mathrm{fm}^{-1}$.

Fig. 3 shows a set of approximations. Similar to [19], these approximations are normalized about the point $p=11.276 \mathrm{fm}^{-1}$. In Fig. 3, the labelled "QCD" corresponds to the application of formula (14). The parameters for (14) are selected from paper [44] for the case $F_{N}^{2} \rightarrow G_{E p}^{2}$ as follows: $N=0.16 ; \Lambda=0.20 \mathrm{GeV}$. Here the proton electric FF was written as (values $0.129 p^{2}$ in dimension $\left.\left[(\mathrm{GeV} / \mathrm{c})^{2}\right]\right)$

$G_{E p}=\frac{1}{\left(1+p^{2} / m_{D}^{2}\right)^{2}}\left(1-0.129 p^{2}\right)$ at $m_{D}^{2}=0.71 G e V^{2}$.

In Fig. 3 uses the following designations for approximations: NRIA [47] as $A \sim p^{-16}$; approximation for pQCD [48, 19] as $p^{-20}$; analytical result (26) for DWFs (22) with DFF in form $p^{-14}$; NRIA [46] as $p^{-12}$; "Approx2" described above; the approximation $A=p^{-2 m}$ at $m=8.7$ [19] is labelled as $p^{-17.4}$; the designation "Global fit" corresponds to the approximation of the experimental data for the momentum interval $p=4.00874-12.36678 \mathrm{fm}^{-1}$ with the resultant function 


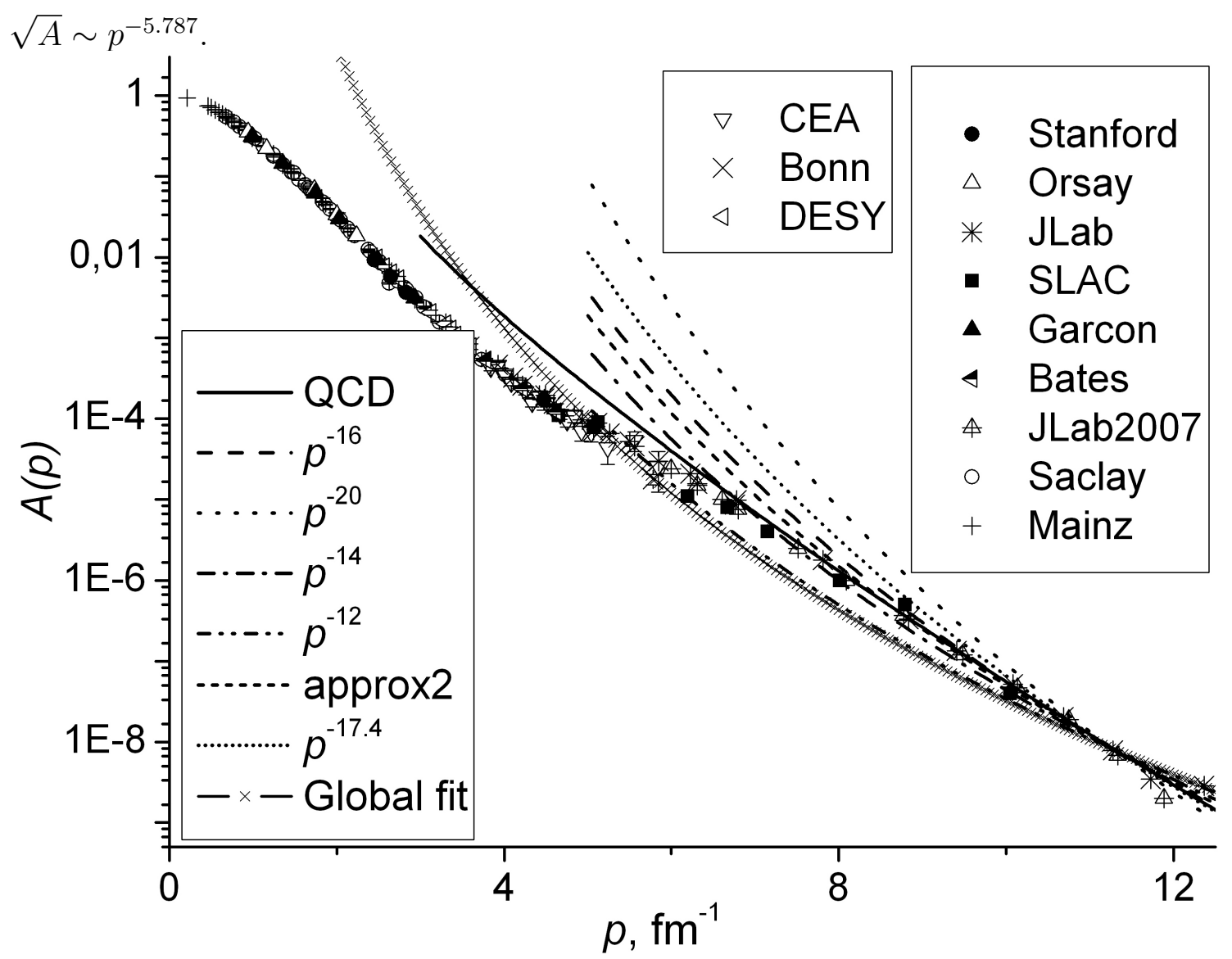

Fig. 3. Approximations of the electric structure function $A(p)$. Approximations and global fit are compared with the experimental data from [30, 31, 32, 16, 17, 33, 34, 35, 36, 37, 38, 3, 2, 4, 38]

So, we can draw the following conclusions:

1. Theoretical features of the estimation of the asymptotic behaviour of the electric structure function $A(p)$ at large momentums are analysed. Asymptotic values of $A(p)$ were obtained for Reid93 potential when using different models and approximations for nucleon FFs. The approximation of the values $A(p)$ in the range momentums at $p=10.6-12.5 \mathrm{fm}^{-1}$ showed, that closer to the experimental approximation are the values for RHOM and VMDM.

2. For electric structure function, the basic theoretical forms of asymptotic behaviour and approximations according to the experimental data and in comparison with the experiment of the leading collaborations are demonstrated. In addition to the QCD approach, the asymptotic for $A(p)$ is represented in the form of the power function $p^{-n}$.

3. The asymptotic behaviour of $A(p)(26)$ has been determined by taking into account the asymptotic behaviour of deuteron FFs 25 and DFF for nucleon FFs. The exact value of $A(p)$ at large momentum values is determined by the asymptotics of the deuteron FFs $G_{i}$, the coefficients of the analytical forms of the DWs and the nucleon isoscalar FFs.

4. The following numerical calculations of the ratio $B / A$ for momentums up $p=12 \mathrm{fm}^{-1}[39$, [65], the tensor $t_{2 j}$ and vector $t_{1 i}$ polarizations [5], the spin correlation coefficients and tensor asymmetries [6] and other polarization observables taking into account the behaviour of the electric structure function are promising.

\section{Література}

[1] D.K. Hasell et al., Annu. Rev. Nucl. Part. Sci. 61, 409 (2011). 
[2] L.C. Alexa et al., Phys. Rev. Lett. 82, 1374 (1999).

[3] D. Abbott et al., Phys. Rev. Lett. 82, 1379 (1999).

[4] R. Alarcon et al., Jefferson Lab PAC33 Proposal December 2007 PR-08-019.

[5] R. Gilman, F. Gross, J. Phys. G. 28, R37 (2002).

[6] G.I. Gakh, A.G. Gakh, E. Tomasi-Gustafsson, Phys. Rev. C 90, 064901 (2014).

[7] D.R. Phillips, S.J. Wallace, N.K. Devine, Phys. Rev. C 72, 014006 (2005).

[8] M. Kohl, Nucl. Phys. A 805, 361 (2008).

[9] M. Piarulli et al., Phys. Rev. C 87, 014006 (2013).

[10] N.A. Khokhlov, A.A. Vakulyuk, Phys. Atom. Nucl., 78, 92 (2015).

[11] T. Gutsche, V.E. Lyubovitskij, I. Schmidt, Phys. Rev. D 94, 116006 (2016).

[12] V.G.J. Stoks et al., Phys. Rev. C 49, 2950 (1994).

[13] J.J. de Swart et al., Few-Body Syst. Suppl. 8, 438 (1995).

[14] V. I. Zhaba, Mod. Phys. Lett. A 31, 1650139 (2016).

[15] D. Abbott et al., Phys. Rev. Lett. 84, 5053 (2000).

[16] J.E. Elias et al., Phys. Rev. 177, 2075 (1969).

[17] S. Galster et al., Nucl. Phys. B 32, 221 (1971).

[18] T.W. Donnelly, A.S. Raskin, Ann. Phys. (N. Y.) 169, 247 (1986).

[19] M. Garcon, J.W. van Orden, Adv. Nucl. Phys. 26, 293 (2001).

[20] M.N. Rosenbluth, Phys. Rev. 79, 615 (1950).

[21] M. Gourdin, Nuovo Cimento, 28, 533 (1963).

[22] F. Gross, Phys. Rev. 136, B140 (1964).

[23] M. Gari, H. Hyuga, Nucl. Phys. A 264, 409 (1976).

[24] N.J. McGurk, H. Fiedeldey, Nucl. Phys. A, 281, 310(1977).

[25] E.L. Lomon, Ann. Phys. 125, 309 (1980).

[26] C. Adamuscín et al., Phys. Rev. C 78, 025202 (2008).

[27] M.I. Haftel, L. Mathelitsch, H.F.K. Zingl, Phys. Rev. C 22, 1285 (1980).

[28] A. Bekzhanov et al., Nucl. Phys. B (Proc. Suppl.) 245, 65 (2013).

[29] A.F. Krutov, Theor. Phys. 3, 5 (2002).

[30] C.D. Buchanan, M.R. Yearian, Phys. Rev. Lett. 15, 303 (1965). 
[31] D. Benaksas et al., Phys. Rev. 148, 1327 (1966).

[32] B. Grossetete et al., Phys. Rev. 141, 1425 (1966).

[33] R. Arnold et al., Phys. Rev. Lett. 35, 776 (1975).

[34] F. Martin et al., Phys. Rev. Lett. 38, 1320 (1977).

[35] G.G. Simon et al., Nucl. Phys. A 364, 285 (1981).

[36] R. Cramer, M. Renkhoff, J. Drees et al., Z. Phys. C 29, 513 (1985).

[37] S. Platchkov et al., Nucl. Phys. A 510, 740 (1990).

[38] M. Garcon et al., Phys. Rev. C 49, 2516 (1994).

[39] S.J. Brodsky, J.R. Hiller, Phys. Rev. D 46, 2141 (1992).

[40] G. Salme, F. M. Lev, E. Pace, Few-Body Syst. Suppl. 12, 235 (2000).

[41] F.M. Lev, E. Pace, G. Salme, Phys. Rev. C 62, 064004 (2000).

[42] S.J. Brodsky, B.T. Chertok, Phys. Rev. D 14, 3003 (1976).

[43] S.J. Brodsky, C.-R. Ji, G.P. Lepage, Phys. Rev. Lett. 51, 83 (1983).

[44] M.P. Rekalo, E. Tomasi-Gustafsson, Eur. Phys. J. A 16, 563 (2003).

[45] A.F. Krutov, V.E. Troitsky, N.A. Tsirova, Theor. Phys. 5, 17 (2004).

[46] A.F. Krutov, V.E. Troitsky, N.A. Tsirova, Theor. Phys. 6, 71 (2005).

[47] A.F. Krutov, V.E. Troitsky, N.A. Tsirova, Vestnik SamSU, Natural Scie. Ser. 3(43), 100 (2006).

[48] J.W. van Orden. Electron Scattering (Kluwer Academic/Plenum Publishers, New York, 2005) 279-289 p.

[49] T. Gutsche et al., Phys. Rev. D 91, 114001 (2015).

[50] V.I. Zhaba, e-print arXiv:nucl-th/1603.05174

[51] A.V. Bekzhanov, S.G. Bondarenko, V.V. Burov, JETP Lett. 99, 613 (2014).

[52] V.V. Burov et al., Europhys. Lett. 24, 443 (1993).

[53] F. Iachello, A.D. Jackson, A. Lande, Phys. Lett. 43B, 191 (1973).

[54] M. Gari, W. Krumpelmann, Z. Phys. A 322, 689 (1985).

[55] J.J. Kelly, Phys. Rev. C 70, 068202 (2004)

[56] R. Bradford et al., Nucl. Phys. B (Proc. Suppl.) 159, 127 (2006)

[57] V.I. Zhaba, Visnyk Lviv Univ. Ser. Phys. 56, 43 (2019).

[58] Y. Huang, W.N. Polyzou, Phys. Rev. C 80, 025503 (2009). 
[59] E. Hummel, J.A. Tjon, Phys. Rev. C 49, 21 (1994).

[60] T.W. Allen, W.H. Klink, W.N. Polyzou, Phys. Rev. C 63, 034002 (2001).

[61] H. Arenhovel, F. Ritz, T. Wilbois, Phys. Rev. C 61, 034002 (2000).

[62] D.R. Phillips, Few-Body Syst. Suppl. 12, 229 (2000).

[63] W. Plessas et al., Few-Body Syst. Suppl. 9, 429 (1995).

[64] S.J. Brodsky, G.R. Farrar, Phys. Rev. D 11, 1309 (1975).

[65] R.J. Holt, R. Gilman, Rep. Prog. Phys. 75, 086301 (2012). 